Bundesgesundheitsbl 2014 · 57:830-839

DOI 10.1007/s00103-014-1982-8

c) Springer-Verlag Berlin Heidelberg 2014
T. Lampert • B. Kuntz · KiGGS Study Group

Abteilung für Epidemiologie und Gesundheitsmonitoring, Robert Koch-Institut, Berlin

\title{
Tabak- und Alkoholkonsum bei 11- bis 17-jährigen Jugendlichen
}

\section{Ergebnisse der KiGGS-Studie - Erste Folgebefragung (KiGGS Welle 1)}

gravierende gesundheitliche Schäden verursachen $[10,11]$. Je früher der Einstieg in den Tabak- und Alkoholkonsum erfolgt, desto größer ist zudem die Wahrscheinlichkeit, im späteren Leben regelmäßig zu rauchen, übermäßig Alkohol zu trinken und eine Tabak- bzw. Alkoholabhängigkeit zu entwickeln [12-14].

Eine wichtige Voraussetzung für die Entwicklung, Umsetzung und Evaluation von Maßnahmen und Programmen der Suchtprävention sind regelmäßig verfügbare und im Hinblick auf ihre Validität, Reliabilität und Repräsentativität belastbare Daten, die Auskunft sowohl über die aktuelle Verbreitung als auch über zeitliche Entwicklungen und Trends des Tabak- und Alkoholkonsums von Jugendlichen geben. Neben den Repräsentativerhebungen der Bundeszentrale für gesundheitliche Aufklärung (BZgA) [15], der Europäischen Schülerstudie zu Alkohol und anderen Drogen (ESPAD) des Instituts für Therapieforschung [16] und der WHOStudie Health Behaviour in School-aged Children (HBSC) $[17,18]$ werden diese Anforderungen auch von der Studie zur Gesundheit von Kindern und Jugendlichen in Deutschland (KiGGS) des Robert Koch-Instituts (RKI) erfüllt. Die Basiserhebung der KiGGS-Studie wurde im Zeitraum von 2003 bis 2006 durchgeführt [19]. Mit der ersten Folgebefragung der KiGGS-Studie (KiGGS Welle 1), die in den Jahren 2009 bis 2012 stattfand, können nun die Ergebnisse der Basiserhebung fortgeschrieben werden. Im Folgenden werden anhand dieser Daten aktuelle Querschnittbefunde zum Tabak- und Alkoholkonsum von 11- bis 17-jährigen
Mädchen und Jungen berichtet. Neben alters- und geschlechtsspezifischen Unterschieden in den Konsumgewohnheiten wird dabei auch auf Unterschiede nach dem sozioökonomischen Status der Familie eingegangen. Unter Hinzuziehung der Daten der KiGGS-Basiserhebung wird auBerdem berichtet, wie sich der Tabak- und Alkoholkonsum von Jugendlichen in den letzten Jahren verändert hat.

\section{Daten und Methode}

KiGGS ist Bestandteil des Gesundheitsmonitorings des RKI und derzeit als kombinierte Querschnitt- und Kohortenstudie realisiert. Ziele, Konzept und Design von KiGGS sind an anderer Stelle ausführlich beschrieben [20-22]. Für den Altersbereich 0 bis 17 Jahre soll KiGGS wiederholt bundesweit erhobene Prävalenzdaten zur gesundheitlichen Situation der in Deutschland lebenden Kinder und Jugendlichen liefern. Die KiGGSBasiserhebung (2003-2006) umfasste Befragungen, Untersuchungen und Laboranalysen, KiGGS Welle 1 (2009-2012) Befragungen in Form von Telefoninterviews. An der KiGGS-Basiserhebung war eine Querschnittstichprobe von insgesamt 17.641 Probanden im Alter von 0 bis 17 Jahren bei einer Response von 66,6\% beteiligt. Die Einzuladenden wurden in einer geschichteten Zufallsstichprobe von 167 Orten Deutschlands zufällig aus den Melderegistern gezogen [20]. Die Stich-

Die KiGGS Study Group: Die Abteilung Epidemiologie und Gesundheitsmonitoring im Robert Koch-Institut. 
probe von KiGGS Welle 1 bestand zum einen aus einer neuen Querschnittstichprobe 0 - bis 6-Jähriger, die wiederum zufällig aus den Melderegistern der ursprünglichen 167 Studienorte gezogen wurden. Zum anderen wurden die ehemaligen Teilnehmenden der KiGGS-Basiserhebung, die inzwischen 6 bis 24 Jahre alt waren und als geschlossene Kohorte weitergeführt werden, zur Befragung eingeladen. Die Telefoninterviews wurden durch geschultes Studienpersonal im RKI durchgeführt. Zum Anrufmanagement und zur Datenerfassung wurde das Softwareprodukt Voxco Version 5.4.4.5 (Voxco Inc., Montréal QC, Kanada) eingesetzt. Vor Beginn der Studie lagen positive Voten der Ethikkommission der Charité-Universitätsmedizin Berlin und des Bundesbeauftragten für den Datenschutz vor, eine Befragung erfolgte nur nach Information und schriftlicher Einverständniserklärung der Sorgeberechtigten Minderjähriger oder der volljährigen Probanden selbst. Insgesamt nahmen 12.368 Kinder und Jugendliche (6093 Mädchen, 6275 Jungen) in dem für den Querschnitt relevanten Altersbereich von 0 bis 17 Jahren teil, darunter 4455 Ersteingeladene (Response 38,8\%) und 7913 Wiedereingeladene (Response 72,9\%).

Das Rauchverhalten wurde in KiGGS Welle 1 mit mehreren Fragen erhoben, die sich unter anderem auf den aktuellen Tabakkonsum sowie auf die Intensität des Rauchens beziehen. Um die Lebenszeitprävalenz des Tabakkonsums zu erfassen, wurden zunächst alle Jugendlichen im Alter von 11 bis 17 Jahren gefragt: „Hast du schon einmal geraucht?" (Antwortkategorien: „Ja“, „Nein“). Diejenigen, die diese Frage bejahten, wurden als Nächstes gefragt, wie oft sie zurzeit rauchen (Antwortkategorien: „Täglich“, „Mehrmals pro Woche“, „Einmal pro Woche“, „Seltener als einmal pro Woche“, „Gar nicht"). Durch das Zusammenfassen verschiedener Antwortkategorien können die Anteile aktueller Raucher (jeglicher, auch gelegentlicher Tabakkonsum), regelmäßiger Raucher (mindestens einmal pro Woche) und täglicher Raucher bestimmt werden. Alle regelmäßigen Raucher wurden zusätzlich nach der Anzahl der pro Tag bzw. pro Woche gerauchten Zigaretten gefragt sowie nach dem Alter, in dem sie begon- nen haben, regelmäßig Zigaretten zu rauchen.

Da in der KiGGS-Basiserhebung zum größten Teil die gleichen Fragen zum Rauchverhalten gestellt wurden, können für die meisten Indikatoren Aussagen zur zeitlichen Entwicklung in den letzten 6 Jahren getroffen werden [19]. Lediglich für die Lebenszeitprävalenz des Rauchens trifft dies nicht zu.

Der Alkoholkonsum wurde mittels 4 Fragen erhoben. Neben der Einstiegsfrage zur Ableitung der Lebenszeitprävalenz („Hast du schon einmal Alkohol getrunken?“, Antwortkategorien: „Ja“, „Nein“) wurde das aus 3 Fragen bestehende, international etablierte AUDIT-C-Instrument eingesetzt [23]. Hierbei handelt es sich um einen Kurzfragebogen des im Auftrag der Weltgesundheitsorganisation (WHO) entwickelten Alcohol Use Disorders Identification Test (AUDIT) [24, 25]. Das AUDIT-C-Instrument (C steht für consumption) wird zur Erfassung des riskanten Alkoholkonsums und zur Verbreitung des Rauschtrinkens (engl. „binge drinking“) im Rahmen des Gesundheitsmonitorings am RKI bereits in der Studie „Gesundheit in Deutschland aktuell“ (GEDA) und der Studie zur Gesundheit Erwachsener in Deutschland (DEGS1) verwendet [2628]. Ursprünglich für das Screening in der Erwachsenenbevölkerung konzipiert, hat sich das AUDIT-C-Instrument auch für den Einsatz bei Jugendlichen als hilfreich erwiesen [29]. Die 3 Fragen des AUDITC-Instruments lauten:

1. Wie oft nimmst du ein alkoholisches Getränk zu dir? (Antwortkategorien: „Nie“, „Einmal pro Monat oder seltener“, „2- bis 4-mal im Monat“, „2- bis 3-mal pro Woche“, „4-mal pro Woche oder öfter").

2. Wenn du Alkohol trinkst, wie viele alkoholische Getränke trinkst du dann üblicherweise an einem Tag? (Antwortkategorien: „1 bis 2 “, , 3 bis 4 “, , 5 bis 6“, „7 bis 9“, „10 oder mehr alkoholische Getränke“).

3. Wie oft trinkst du 6 oder mehr alkoholische Getränke bei einer Gelegenheit, z. B. auf einer Party? (Antwortkategorien: „Nie“, „Seltener als einmal im Monat“, „Jeden Monat“, „Jede Woche“, „Jeden Tag oder fast jeden Tag“).
$\mathrm{Zu}$ den Fragen wurde jeweils der Hinweis gegeben, dass mit einem alkoholischen Getränk z. B. eine kleine Flasche Bier $(0,33 \mathrm{l})$, ein kleines Glas Wein $(0,125 \mathrm{l})$ oder ein doppelter Schnaps $(0,04$ l) gemeint ist. Entsprechend den Instrumentenvorgaben wurden die Antwortkategorien der 3 Einzelfragen jeweils aufsteigend mit Punktwerten von 0 bis 4 versehen und anschließend aufsummiert [23, 24]. Die Spannweite des auf diese Weise gebildeten Summenscores reicht demnach von 0 bis 12. Von riskantem Alkoholkonsum ist bei einem Summenscore von $\geq 4$ bei Mädchen und $\geq 5$ bei Jungen auszugehen [2729]. Die Definition der Schwellenwerte erfolgte wie bei den RKI-Surveys zu Erwachsenen in Anlehnung an Reinert und Ellen sowie Gual et al. [30, 31]. Ein AUDIT-C-Gesamtwert unterhalb der definierten Schwellenwerte ist für die hier betrachtete Altersgruppe der 11- bis 17-Jährigen jedoch nicht mit risikoarmem Alkoholkonsum gleichzusetzen. Gemäß den Empfehlungen des wissenschaftlichen Kuratoriums der Deutschen Hauptstelle für Suchtfragen (DHS) sollten Jugendliche Alkohol weitgehend meiden; etablierte Grenzwerte für risikoarmen Konsum existieren für sie nicht [32]. Die Verbreitung des Rauschtrinkens wird anhand der dritten Einzelfrage des AUDIT-C-Instruments bestimmt. Von regelmäßigem Rauschtrinken ist im Folgenden die Rede, wenn die Jugendlichen angaben, mindestens einmal im Monat oder häufiger 6 oder mehr alkoholische Getränke bei einer Gelegenheit zu konsumieren. Da in der KiGGS-Basiserhebung anstatt des AUDIT-C-Instruments Trinkmenge und Trinkfrequenz in einer kombinierten Frage differenziert für unterschiedliche Getränkesorten erhoben wurden, können Trendaussagen lediglich im Hinblick auf die Lebenszeitprävalenz des Alkoholkonsums getroffen werden [19].

Im Folgenden werden Prävalenzen mit $95 \%$-Konfidenzintervallen für den Tabak- und Alkoholkonsum unter Berücksichtigung von Unterschieden nach Alter, Geschlecht und sozioökonomischem Status (SES) berichtet. Der SES wird anhand eines Index bestimmt, in den Angaben der Eltern zu ihrer schulischen und beruflichen Ausbildung, ihrer beruflichen Stellung und ihrem Haushaltsnet- 
Bundesgesundheitsbl 2014·57:830-839 DOI 10.1007/s00103-014-1982-8

C) Springer-Verlag Berlin Heidelberg 2014

\section{T. Lampert · B. Kuntz $\cdot$ KiGGS Study Group}

\section{Tabak- und Alkoholkonsum bei 11- bis 17-jährigen Jugendlichen. Ergebnisse der KiGGS-Studie - Erste Folgebefragung (KiGGS Welle 1)}

\section{Zusammenfassung}

Analysiert wird der Tabak- und Alkoholkonsum von Jugendlichen in Deutschland. Neben der aktuellen Situation werden auch zeitliche Entwicklungen und Trends berichtet. Als Datenbasis dient die erste Folgebefragung der Studie zur Gesundheit von Kindern und Jugendlichen in Deutschland (KiGGS Welle 1), die in den Jahren 2009 bis 2012 durchgeführt wurde. Einbezogen wurden alle Mädchen und Jungen im Alter von 11 bis 17 Jahren $(n=5258)$. Die Ergebnisse zeigen, dass aktuell $12,0 \%$ der 11 - bis 17 -jährigen Jugendlichen in Deutschland rauchen. Darin eingeschlossen sind $5,4 \%$ der Jugendlichen, die täglich rauchen. Ein riskanter Alkoholkonsum (AUDIT-C-Gesamtwert) ist bei 15,8\% der Heranwachsenden festzustellen, regelmäßiges Rauschtrinken (mindestens einmal im Monat 6 oder mehr alkoholische Getränke bei einer Gelegenheit) bei $11,5 \%$. Zwischen den Geschlechtern sind in Bezug auf die meisten Indikatoren keine signifikanten Unterschiede festzustellen. Lediglich bei den 14- bis 17-Jährigen zeigt sich, dass Jungen häufiger als Mädchen zu regelmäßigem Rauschtrinken neigen ( 23,1 vs. $16,5 \%$, $p<0,01)$. Bezüglich des Rauchens sind darüber hinaus deutliche Unterschiede nach dem sozioökonomischen Status zu beobachten. Beispielsweise gehören Jugendliche aus Familien mit niedrigem sozioökonomischem Status im Verhältnis zu Jugendlichen aus der hohen Statusgruppe signifikant häufiger zu den regelmäßigen und zu den täglichen Rauchern $(\mathrm{OR}=1,95,95 \%-\mathrm{Kl}=1,16-$ $3,27$ bzw. OR=3,71, $95 \%-K I=2,05-6,69)$. Der Zusammenhang zwischen dem sozioökonomischen Status und dem Alkoholkonsum ist schwächer ausgeprägt. Signifikante Unterschiede zeichnen sich lediglich in der Lebenszeitprävalenz des Alkoholkonsums ab, und diese sprechen zudem für einen geringeren
Konsum in der niedrigen im Vergleich zur hohen Statusgruppe $(\mathrm{OR}=0,47,95 \%-\mathrm{KI}=0,33-$ $0,68)$. Unter Hinzuziehung der etwa 6 Jahre zuvor gewonnenen Daten der KiGGS-Basiserhebung kann die Aussage getroffen werden, dass sich die Raucherquote von $20,4 \%$ auf $12,0 \%$ fast halbiert hat. Auch der Anteil der Jugendlichen, die jemals Alkohol getrunken haben, ist von $62,8 \%$ auf $54,4 \%$ deutlich zurückgegangen. Diese Ergebnisse stehen im Einklang mit den Befunden anderer Studien zum Tabak- und Alkoholkonsum von Jugendlichen und sind, insbesondere mit Blick auf den Tabakkonsum, im Zusammenhang mit den in den letzten Jahren verstärkten Präventionsbemühungen zu sehen.

\section{Schlüsselwörter}

Rauchen · Tabakkonsum · Alkoholkonsum . Sozioökonomischer Status · Jugendliche . Gesundheitssurvey

\section{Tobacco and alcohol consumption among 11- to 17-year-old adolescents. Results of the KiGGS study: first follow-up (KiGGS Wave 1)}

\section{Abstract}

In this paper, tobacco and alcohol consumption among adolescents in Germany was analyzed. In addition to the current situation, we report temporal developments and trends. Data were obtained from the first followup of the KiGGS study (KiGGS Wave 1) conducted from 2009 to 2012. All girls and boys aged $11-17$ years $(n=5,258)$ were included. The results show that currently $12.0 \%$ of 11- to 17-year-old adolescents in Germany smoke, $5.4 \%$ of them on a daily basis. Atrisk drinking (AUDIT-C total score) was prevalent among $15.8 \%$ of adolescents, heavy episodic drinking (six or more alcoholic standard drinks on a single occasion at least once a month) among $11.5 \%$. No significant gender differences were found for most indica- tors. However, among adolescents aged 1417 years, boys revealed a greater inclination toward heavy episodic drinking than girls did ( 23.1 vs. $16.5 \%, p<0.01)$. Regarding smoking distinct socioeconomic differences were observed. For example, adolescents from families with a low socioeconomic status (SES) smoke significantly more often on a regular or daily basis compared with their peers from high-SES families $(\mathrm{OR}=1.95,95 \% \mathrm{Cl}=1.16$ 3.27 and $\mathrm{OR}=3.71,95 \% \mathrm{Cl}=2.05-6.69$, respectively). The relationship between SES and alcohol consumption is rather weak. Significant differences emerged only regarding lifetime prevalence of alcohol consumption, and indicate lower consumption rates among low-SES compared with high-SES adolescents
( $\mathrm{OR}=0.47,95 \% \mathrm{Cl}=0.33-0.68)$. Consideration of the KiGGS baseline study data (20032006) shows that smoking prevalence has dropped almost by half from 20.4 to $12.0 \%$. The percentage of adolescents who have ever drunk alcohol has decreased from 62.8 to $54.4 \%$. These results are consistent with the findings of other studies on adolescent tobacco and alcohol consumption and should be considered in the context of preventive efforts that have been strengthened in recent years, especially regarding tobacco use.

Keywords Smoking · Tobacco consumption . Alcohol consumption - Socioeconomic status . Adolescents · Health survey toeinkommen (bedarfsgewichtet) eingehen und der eine Einteilung in niedrige, mittlere und hohe Statusgruppe ermöglicht [33]. Bezüglich des Zusammenhangs zwischen dem sozioökonomischen Status und dem Tabak- bzw. Alkoholkonsum werden außerdem Odds Ratios (OR) ausgewiesen, die mittels binär logistischer Regressionen berechnet wurden. Sie sind als Chancenverhältnisse zu interpretieren und bringen zum Ausdruck, um welchen Faktor die Chance für das Auftreten des jeweils betrachteten Outcomes in der niedrigen bzw. mittleren gegenüber der hohen Statusgruppe, die als Referenzkategorie definiert wurde, erhöht ist.

Alle Analysen wurden mit einem $\mathrm{Ge}$ wichtungsfaktor durchgeführt, der Ab- weichungen der Stichprobe von der Bevölkerungsstruktur (Stand 31.12.2010) hinsichtlich Alter, Geschlecht, Region, Staatsangehörigkeit, Gemeindetyp und Bildungsstand des Haushaltsvorstandes (Mikrozensus 2009) korrigiert. Ferner wurde für die ehemaligen Teilnehmerinnen und Teilnehmer der KiGGSBasiserhebung die unterschiedliche Wie- 


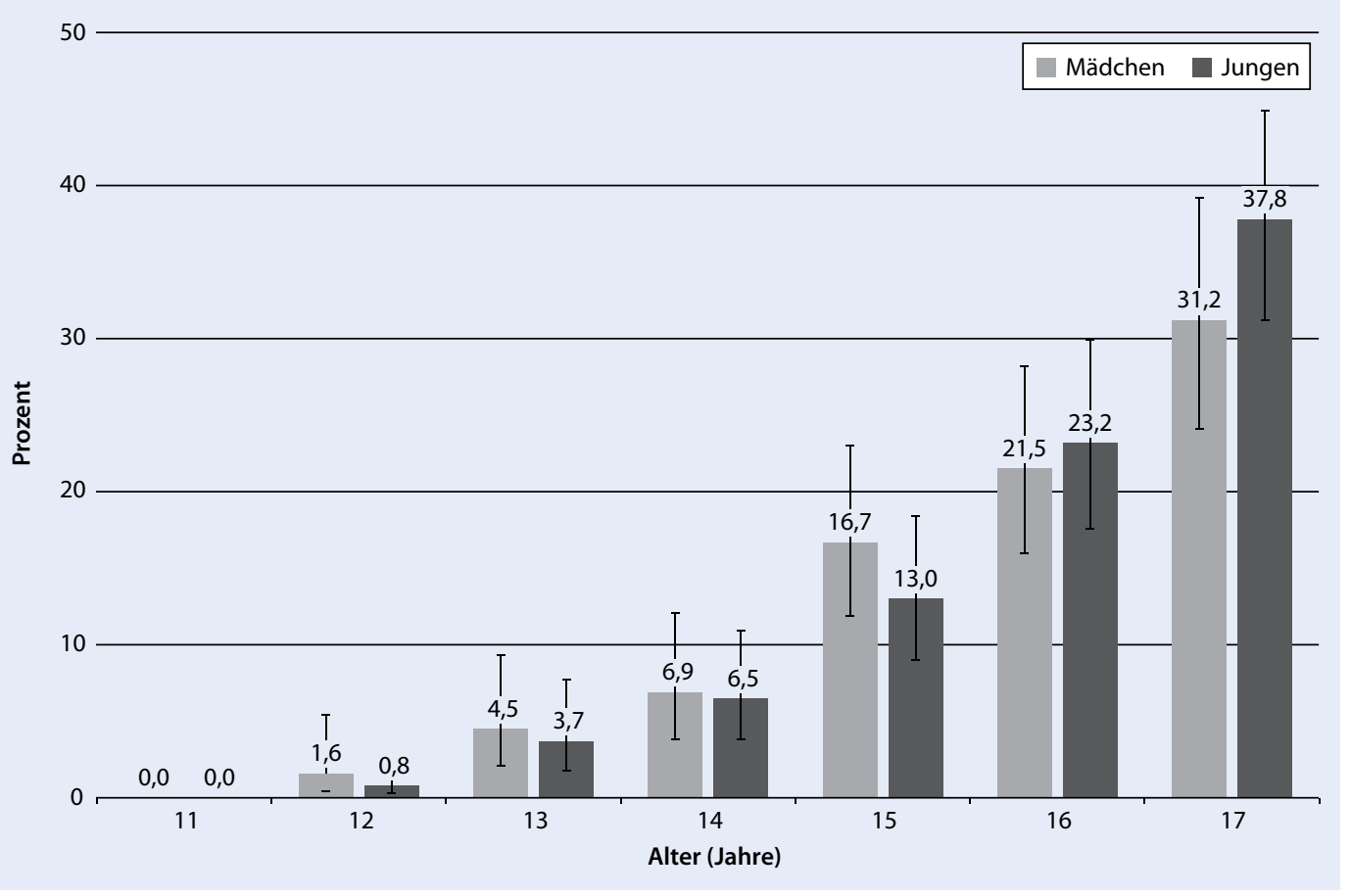

Abb. $1 \varangle$ Anteil der 11-bis 17-jährigen Mädchen und Jungen, die aktuell rauchen $\left(n_{\text {gesamt }}=4944\right)$ derteilnahmebereitschaft mittels Gewichtung nach relevanten Merkmalen aus der KiGGS-Basiserhebung ausgeglichen. Für die Berechnung der Trendanalysen wurden auch die Daten der Basiserhebung bezüglich der oben genannten Merkmale neu gewichtet und auf den Bevölkerungsstand zum 31.12.2010 altersstandardisiert. Details der Methodik von KiGGS Welle 1 sind an anderer Stelle ausführlich beschrieben [34].

Um sowohl die Gewichtung als auch die Korrelation der Teilnehmenden innerhalb einer Gemeinde zu berücksichtigen, wurden die Konfidenzintervalle und $p$ Werte mit Verfahren für komplexe Stichproben berechnet. Gruppenunterschiede/ Trendeffekte wurden mit dem nach RaoScott über die F-Verteilung korrigierten Chi-Quadrat-Test für komplexe Stichproben auf Signifikanz geprüft. Unterschiede werden als statistisch signifikant angesehen, wenn sich die Konfidenzintervalle nicht überschneiden bzw. die Irrtumswahrscheinlichkeit $(p)$ einen Wert kleiner als 0,05 annimmt. Zum Einsatz kam das Softwareprodukt IBM SPSS Statistics Version 20 (IBM Corp., Armonk NY, USA).

\section{Ergebnisse}

Nach den Daten aus KiGGS Welle 1 rauchen 12,0\% der 11- bis 17-jährigen Jugendlichen, wobei keine signifikanten Unterschiede zwischen Mädchen und Jungen bestehen (11,9 vs. 12,1\%). Die Prävalenz liegt bis zum Alter von 13 Jahren unter $5 \%$ und steigt dann sukzessive bis auf 31,2\% bei den 17-jährigen Mädchen und $37,8 \%$ bei den gleichaltrigen Jungen an (• Abb. 1).

Dass sie jemals geraucht haben, trifft auf insgesamt 27,0\% der Jugendlichen im Alter von 11 bis 17 Jahren zu. Der Anteil der regelmäßigen Raucher (mindestens einmal in der Woche) beträgt 7,4\%, der Anteil der täglichen Raucher 5,4\%. Rund 2,3\% der Jugendlichen sind als starke Raucher einzustufen, gemessen an einem Konsum von 10 oder mehr Zigaretten am Tag. Der durchschnittliche Konsum von Jugendlichen, die mindestens einmal in der Woche rauchen, lässt sich mit knapp 7 Zigaretten täglich beziffern. Die alters- und geschlechtsspezifische Betrachtung macht deutlich, dass der Tabakkonsum etwa ab dem 14. Lebensjahr eine weite Verbreitung unter den Heranwachsenden erfährt. Es bestehen bezüglich der betrachteten Indikatoren keine signifikan- ten Unterschiede zwischen Mädchen und Jungen (• Tab. 1).

Um Aussagen über das Einstiegsalter treffen zu können, werden im Folgenden nur die Jugendlichen im Alter von 17 Jahren betrachtet, die regelmäßig, d. h. mindestens einmal in der Woche, rauchen. Im Durchschnitt haben diese im Alter von 15,1 Jahren mit dem regelmäßigen Rauchen begonnen (Mädchen: 15,0 Jahre, Jungen: 15,1 Jahre). Bis zum Alter von 12 Jahren haben lediglich 0,4\% der Mädchen und 4,5\% der Jungen angefangen, regelmäßig zu rauchen. Im Alter von 13 Jahren kommt es dann zu einem ersten sprunghaften Anstieg auf 17,3\% bei den Mädchen und $8,8 \%$ bei den Jungen. Im Alter von 15 Jahren haben 51,8\% der Mädchen und 52,6\% der Jungen, die bei der Befragung 17 Jahre alt waren und angaben, mindestens einmal in der Woche zu rauchen, mit dem regelmäßigen Konsum angefangen. Die andere Hälfte hat im Alter von 16 oder 17 Jahren mit dem regelmäßigen Rauchen begonnen ( $\bullet$ Abb. 2).

Bezüglich des Tabakkonsums von Jugendlichen sind Unterschiede nach dem sozioökonomischen Status festzustellen, insbesondere beim regelmäßigen und täglichen Rauchen. Betrachtet man die nach Alter und Geschlecht adjustierten Odds Ratios, dann lässt sich unter ande- 


\section{Leitthema}

Tab. 1 Tabakkonsum bei 11- bis 17-jährigen Jugendlichen nach Alter und Geschlecht (Prozent, 95\%-Konfidenzintervall)

\begin{tabular}{|c|c|c|c|c|c|c|c|c|c|}
\hline & \multicolumn{3}{|l|}{ Mädchen } & \multicolumn{3}{|l|}{ Jungen } & \multicolumn{3}{|l|}{ Gesamt } \\
\hline & $\begin{array}{l}11 \text { bis } \\
13 \text { Jahre }\end{array}$ & $\begin{array}{l}14 \text { bis } \\
17 \text { Jahre }\end{array}$ & Gesamt & $\begin{array}{l}11 \text { bis } \\
13 \text { Jahre }\end{array}$ & $\begin{array}{l}14 \text { bis } \\
17 \text { Jahre }\end{array}$ & Gesamt & $\begin{array}{l}11 \text { bis } \\
13 \text { Jahre }\end{array}$ & $\begin{array}{l}14 \text { bis } \\
17 \text { Jahre }\end{array}$ & Gesamt \\
\hline & $\begin{array}{l}\% \\
(95 \%-K I)\end{array}$ & $\begin{array}{l}\% \\
(95 \%-\mathrm{KI})\end{array}$ & $\begin{array}{l}\% \\
(95 \%-K I)\end{array}$ & $\begin{array}{l}\% \\
(95 \%-K I)\end{array}$ & $\begin{array}{l}\% \\
(95 \%-K I)\end{array}$ & $\begin{array}{l}\% \\
(95 \%-\mathrm{KI})\end{array}$ & $\begin{array}{l}\% \\
(95 \%-K I)\end{array}$ & $\begin{array}{l}\% \\
(95 \%-\mathrm{KI})\end{array}$ & $\begin{array}{l}\% \\
(95 \%-K I)\end{array}$ \\
\hline $\begin{array}{l}\text { Jemalsraucher } \\
\left(n_{\text {gesamt }}=4945\right)\end{array}$ & $\begin{array}{l}4,9 \\
(3,4-7,1) \\
\end{array}$ & $\begin{array}{l}43,2 \\
(39,3-47,2)\end{array}$ & $\begin{array}{l}26,9 \\
(24,5-29,4) \\
\end{array}$ & $\begin{array}{l}9,2 \\
(6,9-12,2)\end{array}$ & $\begin{array}{l}41,0 \\
(37,4-44,6)\end{array}$ & $\begin{array}{l}27,2 \\
(24,8-29,7) \\
\end{array}$ & $\begin{array}{l}7,1 \\
(5,7-8,8) \\
\end{array}$ & $\begin{array}{l}42,1 \\
(39,4-44,8) \\
\end{array}$ & $\begin{array}{l}27,0 \\
(25,3-28,8) \\
\end{array}$ \\
\hline $\begin{array}{l}\text { Aktuelle Raucher (zu- } \\
\text { mindest gelegentlich) } \\
\left(n_{\text {gesamt }}=4944\right)\end{array}$ & $\begin{array}{l}2,1 \\
(1,1-4,0)\end{array}$ & $\begin{array}{l}19,1 \\
(16,4-22,2)\end{array}$ & $\begin{array}{l}11,9 \\
(10,2-13,8)\end{array}$ & $\begin{array}{l}1,5 \\
(0,8-2,9)\end{array}$ & $\begin{array}{l}20,2 \\
(17,5-23,3)\end{array}$ & $\begin{array}{l}12,1 \\
(10,5-14,0)\end{array}$ & $\begin{array}{l}1,8 \\
(1,1-2,9)\end{array}$ & $\begin{array}{l}19,7 \\
(17,8-21,8)\end{array}$ & $\begin{array}{l}12,0 \\
(10,8-13,3)\end{array}$ \\
\hline $\begin{array}{l}\text { Regelmäßige Raucher } \\
\text { (mindestens einmal pro } \\
\text { Woche) }\left(n_{\text {gesamt }}=4944\right)\end{array}$ & $\begin{array}{l}0,7 \\
(0,2-2,5)\end{array}$ & $\begin{array}{l}12,4 \\
(10,1-15,1)\end{array}$ & $\begin{array}{l}7,4 \\
(6,1-9,0)\end{array}$ & $\begin{array}{l}0,9 \\
(0,4-2,2)\end{array}$ & $\begin{array}{l}12,5 \\
(10,3-15,1)\end{array}$ & $\begin{array}{l}7,5 \\
(6,2-9,0)\end{array}$ & $\begin{array}{l}0,8 \\
(0,4-1,7)\end{array}$ & $\begin{array}{l}12,5 \\
(10,9-14,2)\end{array}$ & $\begin{array}{l}7,4 \\
(6,5-8,5)\end{array}$ \\
\hline $\begin{array}{l}\text { Tägliche Raucher } \\
\left(n_{\text {gesamt }}=4944\right)\end{array}$ & $\begin{array}{l}0,5 \\
(0,1-2,7)\end{array}$ & $\begin{array}{l}8,9 \\
(7,0-11,3)\end{array}$ & $\begin{array}{l}5,3 \\
(4,2-6,8)\end{array}$ & $\begin{array}{l}0,8 \\
(0,3-2,1) \\
\end{array}$ & $\begin{array}{l}9,2 \\
(7,3-11,5)\end{array}$ & $\begin{array}{l}5,5 \\
(4,4-6,9) \\
\end{array}$ & $\begin{array}{l}0,7 \\
(0,3-1,6)\end{array}$ & $\begin{array}{l}9,1 \\
(7,8-10,5)\end{array}$ & $\begin{array}{l}5,4 \\
(4,7-6,3)\end{array}$ \\
\hline $\begin{array}{l}\text { Starke Raucher }(10+\text { Zi- } \\
\text { garetten pro Tag }) \\
\left(n_{\text {gesamt }}=4943\right)\end{array}$ & $\begin{array}{l}0,0 \\
(0,0-0,3)\end{array}$ & $\begin{array}{l}3,4 \\
(2,2-5,2)\end{array}$ & $\begin{array}{l}2,0 \\
(1,3-3,0)\end{array}$ & $\begin{array}{l}0,1 \\
(0,0-0,8)\end{array}$ & $\begin{array}{l}4,6 \\
(3,2-6,4)\end{array}$ & $\begin{array}{l}2,6 \\
(1,9-3,7)\end{array}$ & $\begin{array}{l}0,1 \\
(0,0-0,4)\end{array}$ & $\begin{array}{l}4,0 \\
(3,0-5,2)\end{array}$ & $\begin{array}{l}2,3 \\
(1,8-3,0)\end{array}$ \\
\hline $\begin{array}{l}\text { Täglich gerauchte Zigaret- } \\
\text { ten }{ }^{\mathrm{a}}(\text { Mittelwert }(95 \%-\mathrm{KI})) \\
\left(n_{\text {gesamt }}=346\right)\end{array}$ & $\begin{array}{l}4,5 \\
(3,1-5,9)\end{array}$ & $\begin{array}{l}6,5 \\
(5,1-7,9)\end{array}$ & $\begin{array}{l}6,4 \\
(5,1-7,7)\end{array}$ & $\begin{array}{l}2,6 \\
(0,5-4,8)\end{array}$ & $\begin{array}{l}7,1 \\
(6,0-8,2)\end{array}$ & $\begin{array}{l}6,8 \\
(5,8-7,9)\end{array}$ & $\begin{array}{l}3,4 \\
(1,8-5,1)\end{array}$ & $\begin{array}{l}6,8 \\
(5,9-7,7)\end{array}$ & $\begin{array}{l}6,6 \\
(5,8-7,5)\end{array}$ \\
\hline
\end{tabular}

Tab. 2 Tabakkonsum bei 11- bis 17-jährigen Mädchen und Jungen nach sozioökonomischem Status. Prävalenzen (\%) und mit binär logistischen Regressionen berechnete altersadjustierte Odds Ratios (OR) mit 95\%-Konfidenzintervallen (95\%-KI) und p-Werten

\begin{tabular}{|c|c|c|c|c|c|c|}
\hline & \multicolumn{2}{|c|}{ Rauchen (aktuell) $\left(n_{\text {gesamt }}=4882\right)$} & \multicolumn{2}{|c|}{ Rauchen (regelmäßig) $\left(n_{\text {gesamt }}=4882\right)$} & \multicolumn{2}{|c|}{ Rauchen (täglich) $\left(n_{\text {gesamt }}=4882\right)$} \\
\hline & $\%(95 \%-K I)$ & $\begin{array}{l}\text { OR }(95 \%-\mathrm{KI}) \\
p \text {-Wert }\end{array}$ & $\%(95 \%-K I)$ & $\begin{array}{l}\text { OR }(95 \%-\mathrm{KI}) \\
p \text {-Wert }\end{array}$ & $\%(95 \%-K I)$ & $\begin{array}{l}\text { OR (95\%-KI) } \\
p \text {-Wert }\end{array}$ \\
\hline \multicolumn{7}{|c|}{ Mädchen } \\
\hline \multicolumn{7}{|c|}{ Sozioökonomischer Status } \\
\hline Niedrig & $13,9(9,2-20,5)$ & $\begin{array}{l}1,70(0,90-3,22) \\
p=0,101\end{array}$ & $7,8(4,8-12,5)$ & $\begin{array}{l}1,48(0,70-3,14) \\
p=0,299\end{array}$ & $7,7(4,7-12,3)$ & $\begin{array}{l}7,02(1,87-26,41) \\
p<0,01\end{array}$ \\
\hline Mittel & $12,3(10,1-15,0)$ & $\begin{array}{l}1,64(1,02-2,64) \\
p<0,05\end{array}$ & $7,9(6,0-10,2)$ & $\begin{array}{l}1,68(0,90-3,14) \\
p=0,101\end{array}$ & $5,6(4,0-7,7)$ & $\begin{array}{l}5,41(1,54-19,01) \\
p<0,01\end{array}$ \\
\hline Hoch & $7,5(5,2-10,5)$ & Ref. & $4,5(2,7-7,4)$ & Ref. & $1,0(0,3-3,4)$ & Ref. \\
\hline \multicolumn{7}{|l|}{ Jungen } \\
\hline \multicolumn{7}{|c|}{ Sozioökonomischer Status } \\
\hline Niedrig & $14,8(10,2-20,9)$ & $\begin{array}{l}1,31(0,74-2,35) \\
p=0,355\end{array}$ & $10,7(7,1-16,0)$ & $\begin{array}{l}2,42(1,23-4,75) \\
p<0,05\end{array}$ & $8,5(5,3-13,4)$ & $\begin{array}{l}2,65(1,31-5,36) \\
p<0,01\end{array}$ \\
\hline Mittel & $11,3(9,6-13,3)$ & $\begin{array}{l}1,10(0,75-1,62) \\
p=0,615\end{array}$ & $7,1(5,7-8,9)$ & $\begin{array}{l}1,73(1,04-2,88) \\
p<0,05\end{array}$ & $5,1(3,9-6,6)$ & $\begin{array}{l}1,70(1,01-2,85) \\
p<0,05\end{array}$ \\
\hline Hoch & $10,3(7,9-13,2)$ & Ref. & $4,3(2,8-6,6)$ & Ref. & $3,1(2,0-4,7)$ & Ref. \\
\hline \multicolumn{7}{|l|}{ Gesamt } \\
\hline \multicolumn{7}{|c|}{ Sozioökonomischer Status } \\
\hline Niedrig & $14,4(11,1-18,5)$ & $\begin{array}{l}1,48(1,00-2,19) \\
p=0,052\end{array}$ & $9,4(6,9-12,7)$ & $\begin{array}{l}1,95(1,16-3,27) \\
p<0,05\end{array}$ & $8,1(5,8-11,3)$ & $\begin{array}{l}3,71(2,05-6,69) \\
p<0,001\end{array}$ \\
\hline Mittel & $11,8(10,4-13,4)$ & $\begin{array}{l}1,33(0,97-1,82) \\
p=0,081\end{array}$ & $7,5(6,3-8,9)$ & $\begin{array}{l}1,72(1,10-2,68) \\
p<0,05\end{array}$ & $5,3(4,3-6,5)$ & $\begin{array}{l}2,61(1,57-4,32) \\
p<0,001\end{array}$ \\
\hline Hoch & $8,9(7,1-11,1)$ & Ref. & $4,4(3,1-6,3)$ & Ref. & $2,1(1,4-3,2)$ & Ref. \\
\hline
\end{tabular}

rem die Aussage treffen, dass Jugendliche aus Familien mit niedrigem sozioökonomischem Status 2,0-mal häufiger regelmäBig rauchen als Jugendliche aus Familien mit hohem sozioökonomischem Status.
Bezüglich des täglichen Rauchens beträgt das entsprechende Chancenverhältnis 3,7:1 zuungunsten der Jugendlichen aus der niedrigen Statusgruppe. Statistisch signifikante Unterschiede zeigen sich auch zwischen der mittleren und hohen Statusgruppe, bei Mädchen hinsichtlich des aktuellen und täglichen Rauchens, bei Jungen hinsichtlich des regelmäßigen und täglichen Rauchens (• Tab. 2). 

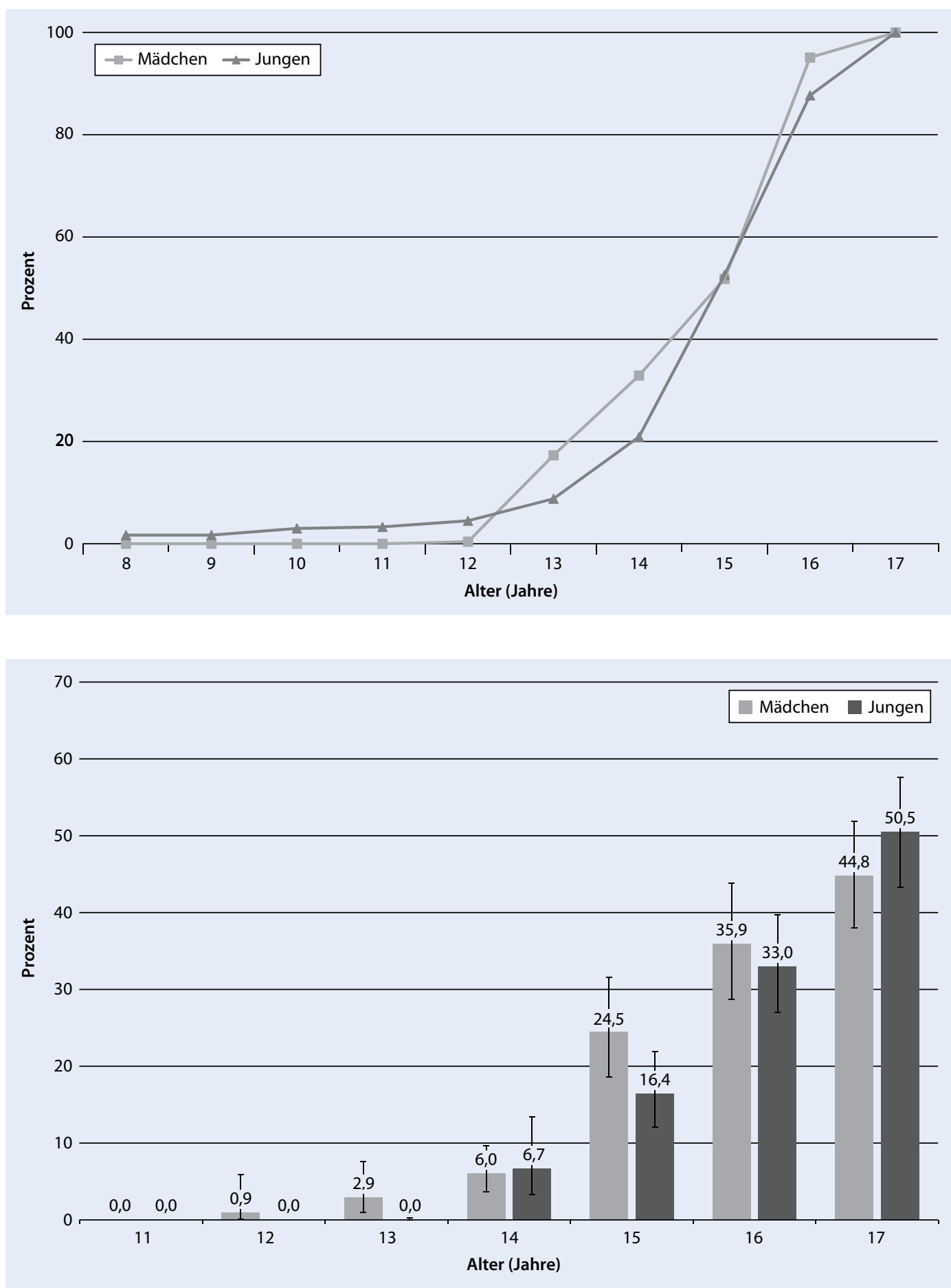

Abb. $2 \triangleleft$ Alter bei Einstieg in den regelmäßigen Tabakkonsum (mindestens einmal pro Woche): kumulative Häufigkeiten auf Basis der Angaben der 17-jährigen Mädchen und Jungen, die zum Zeitpunkt der Befragung mindestens einmal in der Woche rauchen $\left(n_{\text {gesamt }}=153\right)$
Abb. $3<$ Anteil der 11-bis 17-jährigen Mädchen und Jungen mit riskantem Alkoholkonsum (AUDIT-C) $\left(n_{\text {gesamt }}=4909\right)$
Dass sie jemals alkoholische Getränke konsumiert haben, trifft auf insgesamt 54,4\% der 11- bis 17-jährigen Jugendlichen zu. Legt man die Ergebnisse des AUDITC-Gesamtwertes zugrunde, zeigen 15,8\% der Jugendlichen ein riskantes Konsumverhalten. Regelmäßiges Rauschtrinken, bezogen auf den mindestens monatlichen Konsum von 6 und mehr alkoholischen Getränken bei einer Gelegenheit, wird von $11,5 \%$ der Jugendlichen berichtet (• Tab. 3).

Wie beim Rauchen, so sind auch beim Alkoholkonsum nur geringe Unterschiede im Konsumverhalten von Mädchen und Jungen festzustellen. Lediglich bei den 14- bis 17-Jährigen ist zu beobachten, dass Jungen häufiger als Mädchen zu regelmäßigem Rauschtrinken neigen ( 23,1 vs. $16,5 \% ; p<0,01)$. Bei beiden Ge- schlechtern zeichnet sich im Altersgang eine deutliche Zunahme des Alkoholkonsums ab. Mit Blick auf den riskanten Alkoholkonsum steigen die Prävalenzen bis zum 17. Lebensjahr auf $44,8 \%$ bei Mädchen und 50,5\% bei Jungen an (• Abb. 3).

Die statusspezifischen Unterschiede sind beim Alkoholkonsum geringer ausgeprägt als beim Rauchen. Außerdem weisen sie in eine andere Richtung. Dem- 


\section{Leitthema}

Tab. 3 Alkoholkonsum bei 11- bis 17-jährigen Jugendlichen nach Alter und Geschlecht (Prozent, 95\%-Konfidenzintervall)

\begin{tabular}{|c|c|c|c|c|c|c|c|c|c|}
\hline & \multicolumn{3}{|l|}{ Mädchen } & \multicolumn{3}{|l|}{ Jungen } & \multicolumn{3}{|l|}{ Gesamt } \\
\hline & $\begin{array}{l}11 \text { bis } 13 \text { Jah- } \\
\text { re }\end{array}$ & $\begin{array}{l}14 \text { bis } \\
17 \text { Jahre }\end{array}$ & Gesamt & $\begin{array}{l}11 \text { bis } \\
13 \text { Jahre }\end{array}$ & $\begin{array}{l}14 \text { bis } \\
17 \text { Jahre }\end{array}$ & Gesamt & $\begin{array}{l}11 \text { bis } \\
13 \text { Jahre }\end{array}$ & $\begin{array}{l}14 \text { bis } \\
17 \text { Jahre }\end{array}$ & Gesamt \\
\hline & $\begin{array}{l}\% \\
\text { (95\%-KI) }\end{array}$ & $\begin{array}{l}\% \\
\text { (95\%-KI) }\end{array}$ & $\begin{array}{l}\% \\
\text { (95\%-KI) }\end{array}$ & $\begin{array}{l}\% \\
\text { (95\%-KI) }\end{array}$ & $\begin{array}{l}\% \\
\text { (95\%-KI) }\end{array}$ & $\begin{array}{l}\% \\
\text { (95\%-KI) }\end{array}$ & $\begin{array}{l}\% \\
\text { (95\%-KI) }\end{array}$ & $\begin{array}{l}\% \\
\text { (95\%-KI) }\end{array}$ & $\begin{array}{l}\% \\
\text { (95\%-KI) }\end{array}$ \\
\hline $\begin{array}{l}\text { Lebenszeitprävalenz } \\
\left(n_{\text {gesamt }}=4947\right)\end{array}$ & $\begin{array}{l}20,2 \\
(17,3-23,4)\end{array}$ & $\begin{array}{l}80,6 \\
(76,7-83,9) \\
\end{array}$ & $\begin{array}{l}54,8 \\
(52,2-57,5) \\
\end{array}$ & $\begin{array}{l}21,9 \\
(18,3-25,9) \\
\end{array}$ & $\begin{array}{l}78,5 \\
(75,0-81,7) \\
\end{array}$ & $\begin{array}{l}53,9 \\
(51,1-56,8) \\
\end{array}$ & $\begin{array}{l}21,0 \\
(18,7-23,5)\end{array}$ & $\begin{array}{l}79,5 \\
(76,8-82,0) \\
\end{array}$ & $\begin{array}{l}54,4 \\
(52,2-56,5) \\
\end{array}$ \\
\hline $\begin{array}{l}\text { Risikokonsum }^{\mathrm{a}} \\
\left(n_{\text {gesamt }}=4909\right)\end{array}$ & $\begin{array}{l}1,3 \\
(0,5-3,1)\end{array}$ & $\begin{array}{l}27,9 \\
(24,5-31,6)\end{array}$ & $\begin{array}{l}16,5 \\
(14,5-18,8)\end{array}$ & $\begin{array}{l}0,0 \\
(0,0-0,1)\end{array}$ & $\begin{array}{l}26,6 \\
(23,3-30,2)\end{array}$ & $\begin{array}{l}15,1 \\
(13,1-17,3)\end{array}$ & $\begin{array}{l}0,6 \\
(0,3-1,5)\end{array}$ & $\begin{array}{l}27,3(24,5- \\
30,2)\end{array}$ & $\begin{array}{l}15,8 \\
(14,2-17,6)\end{array}$ \\
\hline $\begin{array}{l}\text { Regelmäßiges Rauschtrin- } \\
\operatorname{ken}^{\text {b }}\left(n_{\text {gesamt }}=4943\right)\end{array}$ & $\begin{array}{l}0,9 \\
(0,3-2,8)\end{array}$ & $\begin{array}{l}16,5 \\
(13,8-19,5)\end{array}$ & $\begin{array}{l}9,8 \\
(8,3-11,7)\end{array}$ & $\begin{array}{l}0,3 \\
(0,1-0,7)\end{array}$ & $\begin{array}{l}23,1 \\
(20,0-26,5)\end{array}$ & $\begin{array}{l}13,1 \\
(11,3-15,2)\end{array}$ & $\begin{array}{l}0,6 \\
(0,2-1,4)\end{array}$ & $\begin{array}{l}19,8(17,5- \\
22,2)\end{array}$ & $\begin{array}{l}11,5 \\
(10,2-13,0)\end{array}$ \\
\hline
\end{tabular}

${ }^{a}$ AUDIT-C-Gesamtwert $\geq 4$ (Mädchen) bzw. $\geq 5$ (Jungen)

bRegelmäßiges Rauschtrinken: 6 oder mehr Getränke bei einer Gelegenheit, mindestens einmal im Monat

Tab. 4 Alkoholkonsum bei 11- bis 17-jährigen Jugendlichen nach Sozialstatus. Prävalenzen (\%) und mit binär logistischen Regressionen berechnete altersadjustierte Odds Ratios (OR) mit 95\%-Konfidenzintervallen (95\%-KI) und p-Werten

\begin{tabular}{|c|c|c|c|c|c|c|}
\hline & \multicolumn{2}{|c|}{$\begin{array}{l}\text { Lebenszeitprävalenz } \\
\left(n_{\text {gesamt }}=4885\right)\end{array}$} & \multicolumn{2}{|l|}{$\begin{array}{l}\text { Risikokonsum } \\
\left(n_{\text {gesamt }}=4847\right)\end{array}$} & \multicolumn{2}{|c|}{$\begin{array}{l}\text { Regelmäßiges Rauschtrinken } \\
\left(n_{\text {gesamt }}=4881\right)\end{array}$} \\
\hline & $\%(95 \%-K I)$ & $\begin{array}{l}\text { OR }(95 \%-\mathrm{KI}) \\
p \text {-Wert }\end{array}$ & $\%(95 \%-K I)$ & $\begin{array}{l}\text { OR }(95 \%-\mathrm{KI}) \\
p \text {-Wert }\end{array}$ & $\%(95 \%-\mathrm{KI})$ & $\begin{array}{l}\text { OR }(95 \%-\mathrm{KI}) \\
p \text {-Wert }\end{array}$ \\
\hline \multicolumn{7}{|c|}{ Mädchen } \\
\hline \multicolumn{7}{|c|}{ Sozioökonomischer Status } \\
\hline Niedrig & $49,7(41,5-57,8)$ & $\begin{array}{l}0,48(0,28-0,83) \\
p<0,01\end{array}$ & $15,6(10,5-22,7)$ & $\begin{array}{l}0,80(0,43-1,50) \\
p=0,490\end{array}$ & $8,0(4,4-13,9)$ & $\begin{array}{l}0,85(0,40-1,81) \\
p=0,674\end{array}$ \\
\hline Mittel & $58,4(55,2-61,6)$ & $\begin{array}{l}1,25(0,92-1,70) \\
p=0,145\end{array}$ & $17,7(15,2-20,5)$ & $\begin{array}{l}1,13(0,76-1,67) \\
p=0,548\end{array}$ & $11,3(9,2-13,8)$ & $\begin{array}{l}1,47(0,94-2,31) \\
p=0,092\end{array}$ \\
\hline Hoch & $50,9(45,7-56,1)$ & Ref. & $14,5(11,3-18,2)$ & Ref. & $7,4(5,3-10,3)$ & Ref. \\
\hline \multicolumn{7}{|l|}{ Jungen } \\
\hline \multicolumn{7}{|c|}{ Sozioökonomischer Status } \\
\hline Niedrig & $51,1(42,9-59,3)$ & $\begin{array}{l}\mathbf{0 , 4 7}(0,28-0,78) \\
p<0,01\end{array}$ & $15,1(10,7-20,7)$ & $\begin{array}{l}0,66(0,37-1,19) \\
p=0,166\end{array}$ & $12,9(9,0-18,2)$ & $\begin{array}{l}0,86(0,48-1,52) \\
p=0,593\end{array}$ \\
\hline Mittel & $54,5(51,0-57,9)$ & $\begin{array}{l}0,81(0,61-1,07) \\
p=0,136\end{array}$ & $15,0(12,7-17,7)$ & $\begin{array}{l}0,85(0,56-1,31) \\
p=0,470\end{array}$ & $13,8(11,6-16,2)$ & $\begin{array}{l}1,20(0,78-1,84) \\
p=0,409\end{array}$ \\
\hline Hoch & $57,3(53,5-61,0)$ & Ref. & $16,1(12,6-20,3)$ & Ref. & $12,0(9,0-15,9)$ & Ref. \\
\hline \multicolumn{7}{|l|}{ Gesamt } \\
\hline \multicolumn{7}{|c|}{ Sozioökonomischer Status } \\
\hline Niedrig & $50,5(44,5-56,4)$ & $\begin{array}{l}0,47(0,33-0,68) \\
p<0,001\end{array}$ & $15,3(11,7-19,8)$ & $\begin{array}{l}0,73(0,49-1,09) \\
p=0,127\end{array}$ & $10,7(7,8-14,3)$ & $\begin{array}{l}0,86(0,56-1,32) \\
p=0,491\end{array}$ \\
\hline Mittel & $56,5(54,1-58,9)$ & $\begin{array}{l}0,99(0,80-1,22) \\
p=0,989\end{array}$ & $16,4(14,4-18,5)$ & $\begin{array}{l}0,98(0,73-1,32) \\
p=0,899\end{array}$ & $12,5(10,9-14,4)$ & $\begin{array}{l}1,31(0,94-1,82) \\
p=0,112\end{array}$ \\
\hline Hoch & $54,2(50,9-57,5)$ & Ref. & $15,3(13,0-17,9)$ & Ref. & $9,8(7,9-12,2)$ & Ref. \\
\hline
\end{tabular}

nach ist die statistische Chance, jemals Alkohol getrunken zu haben, bei Jugendlichen aus Familien mit niedrigem sozioökonomischem Status nur etwa halb so hoch wie bei Gleichaltrigen aus der hohen Statusgruppe. Die Unterschiede zeichnen sich dabei erst in der binär logistischen Regressionsanalyse $\mathrm{ab}$, da dort für bestehende Altersunterschiede zwischen Jugendlichen mit niedrigem und hohem sozioökonomischem Status statistisch kontrolliert wird. Mit Blick auf den riskan- ten Alkoholkonsum und das Rauschtrinken zeigt sich ein ähnliches Muster, die Unterschiede zwischen den Statusgruppen sind aber nicht statistisch signifikant (• Tab. 4).

Aussagen über zeitliche Entwicklungen und Trends im Tabak- und Alkoholkonsum von Jugendlichen sind nur für die in der KiGGS-Basiserhebung und KiGGS Welle 1 gleichermaßen erhobenen Indikatoren möglich.
Im Hinblick auf den Tabakkonsum lässt sich feststellen, dass sich der Anteil der aktuell rauchenden Jugendlichen zwischen beiden Erhebungen, also etwa innerhalb von 6 Jahren, von 20,4 auf $12,0 \%$ nahezu halbiert hat $(p<0,001)$. Ein noch deutlicherer Rückgang zeichnet sich beim regelmäßigen und täglichen Tabakkonsum ab (• Tab. 5). Gleichzeitig ist das durchschnittliche Einstiegsalter in den regelmäßigen Tabakkonsum angestiegen, und zwar von 14,2 Jahre auf 15,1 Jahre 


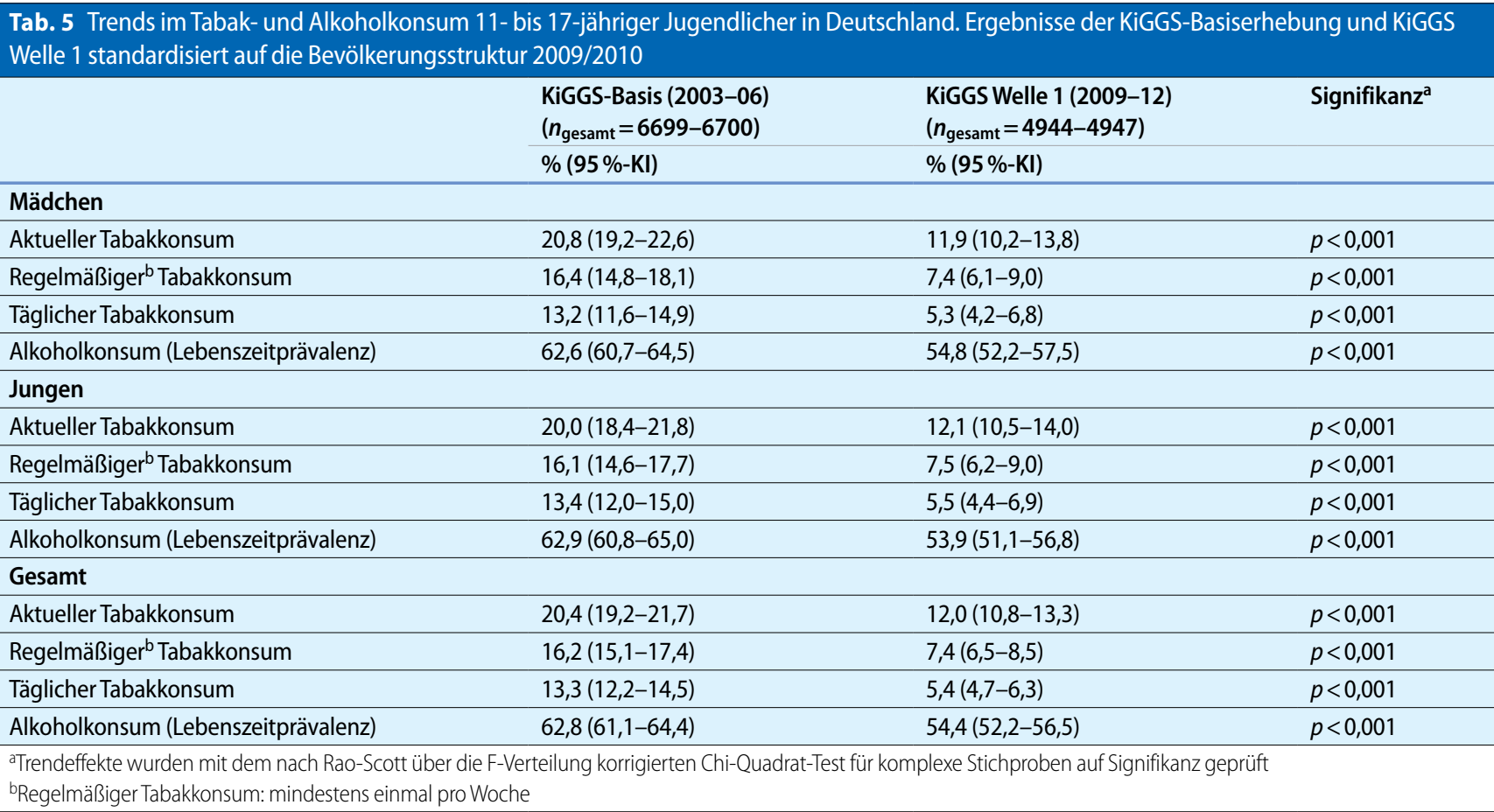

(Mädchen: von 14,2 auf 15,0 Jahre, Jungen: von 14,1 auf 15,1 Jahre).

Veränderungen im Alkoholkonsum lassen sich mit den KiGGS-Daten nur an der Lebenszeitprävalenz festmachen. Der Anteil der 11- bis 17-jährigen Jugendlichen ist demnach zwischen beiden Erhebungen von 62,8 auf $54,4 \%$ gesunken $(p<0,001)$.

\section{Diskussion}

Die Ergebnisse aus KiGGS Welle 1 zeigen, dass aktuell 12,0\% der 11- bis 17-jährigen Jugendlichen in Deutschland rauchen. Darin eingeschlossen sind 5,4\% der Jugendlichen, die täglich rauchen. Ein riskanter Alkoholkonsum (AUDIT-C-Gesamtwert) ist bei 15,8\% der Jugendlichen festzustellen, regelmäßiges Rauschtrinken (mindestens einmal im Monat 6 oder mehr alkoholische Getränke bei einer Gelegenheit) bei 11,5\%. Zwischen Mädchen und Jungen bestehen in Bezug auf die meisten Indikatoren keine signifikanten Unterschiede. Dagegen treten Unterschiede nach dem sozioökonomischen Status zutage, die allerdings beim Tabak- und Alkoholkonsum in eine unterschiedliche Richtung weisen. Bezüglich des Rauchens zeigen sich deutliche Unterschiede zuungunsten von Jugendlichen aus Familien mit niedrigem sozioökonomischem Sta- tus, was sich z. B. an einem höheren Anteil an regelmäßigen und täglichen Rauchern festmachen lässt. Im Gegensatz dazu ist der Zusammenhang zwischen dem sozioökonomischen Status und dem Alkoholkonsum schwächer ausgeprägt. Signifikante Unterschiede zeichnen sich lediglich in der Lebenszeitprävalenz des Alkoholkonsums ab, und diese sprechen zudem für einen geringeren Konsum in der niedrigen im Vergleich zur hohen Statusgruppe. Unter Hinzuziehung der etwa 6 Jahre zuvor erhobenen Daten der KiGGS-Basiserhebung kann die Aussage getroffen werden, dass sich die Raucherquote von 20,4 auf $12,0 \%$ fast halbiert hat. Außerdem wird seltener regelmäßig oder täglich geraucht, und die Jugendlichen, die rauchen, fangen gut $1 \mathrm{Jahr}$ später mit dem Rauchen an. Mit Blick auf den Alkoholkonsum kann die zeitliche Entwicklung nur anhand der Lebenszeitprävalenz betrachtet werden, da der AUDIT-C in der KiGGS-Basiserhebung nicht eingesetzt wurde. Der Anteil der Jugendlichen, die jemals Alkohol getrunken haben, ist danach von 62,8 auf $54,4 \% \mathrm{zu}$ rückgegangen.

Die berichteten Ergebnisse stehen weitgehend im Einklang mit den Befunden anderer Studien zum Tabak- und Alkoholkonsum von Jugendlichen in
Deutschland [15, 16, 18, 35]. So belegen auch die Ergebnisse der Repräsentativerhebungen der BZgA, dass der Anteil der jugendlichen Raucher deutlich zurückgegangen ist. Die Studien der BZgA beziehen sich auf die Altersspanne der 12- bis 17-Jährigen. Zieht man als Vergleichswerte für die KiGGS-Ergebnisse die BZgA-Erhebungen aus den Jahren 2004 und 2010 heran, dann betragen die Raucherquoten 23,5 und $12,9 \%[15,35]$. Die auf Basis der Daten der KiGGS-Basiserhebung und KiGGS Welle 1 für die 12- bis 17-Jährigen ermittelten Prävalenzen fallen mit 23,6 und 13,9\% ganz ähnlich aus. Auch die Daten der HBSC- und ESPAD-Studie, die sich auf Jugendliche im Alter von 11 bis 15 bzw. 15 bis 16 Jahren beziehen, sprechen dafür, dass der Anteil der Raucher unter den Jugendlichen in den letzten Jahren deutlich abgenommen hat $[16,18]$.

Der im Rahmen von KiGGS Welle 1 ermittelte Rückgang der Lebenszeitprävalenz des Alkoholkonsums wird ebenfalls durch die Ergebnisse der BZgA-Repräsentativerhebungen sowie der HBSCund ESPAD-Studie gestützt. Laut BZgA ist die Lebenszeitprävalenz des Alkoholkonsums 12- bis 17-jähriger Jugendlicher im Zeitraum von 2001 bis 2011 von 87,0 auf $72,6 \%$ zurückgegangen [9]. Die 
ESPAD-Daten für den Zeitraum von 2003 bis 2011 sprechen sowohl mit Blick auf die Lebenszeit- als auch auf die 12-Monatsund 30-Tage-Prävalenz des Alkoholkonsums für einen signifikanten Rückgang bei beiden Geschlechtern [16]. Bezüglich der 30-Tage-Prävalenz des Rauschtrinkens bzw. der Verbreitung alkoholbedingter Rauscherfahrungen ist nach den BZgA-Repräsentativerhebungen sowie der HBSC- und ESPAD-Studie ebenfalls von einem deutlichen Rückgang auszugehen $[9,16,18]$.

Der Rückgang des Rauchens bei Jugendlichen ist vor dem Hintergrund der verstärkten politischen Bemühungen um die Eindämmung des Rauchens und die Förderung des Nichtraucherschutzes zu sehen [1, 36-38]. Mit Blick auf den Beobachtungszeitraum ist unter anderem auf mehrere Tabaksteuererhöhungen, die Heraufsetzung der Altersgrenze für den Kauf und Konsum von Tabakprodukten, die Einschränkung bzw. das weitgehende Verbot von Tabakwerbung entsprechend geltendem EU-Recht sowie die seit 2007 erlassenen Nichtraucherschutzgesetze des Bundes und der Länder, die sich auf öffentliche Gebäude und Verkehrsmittel, Schulen und Krankenhäuser sowie auf gastronomische Betriebe beziehen, $\mathrm{zu}$ verweisen. Außerdem hat eine Ausweitung bevölkerungsbezogener Aufklärungskampagnen und settingbezogener Programme zur Suchtprävention stattgefunden sowie eine Veränderung der gesellschaftlichen Einstellungen gegenüber dem Rauchen [1, 36-38]. Auch wenn die genauen Effekte dieser Maßnahmen auf das Rauchverhalten nur schwer zu quantifizieren sind, so wird doch davon ausgegangen, dass sie einen erheblichen Anteil daran haben, dass mittlerweile weniger Jugendliche mit dem Rauchen anfangen [39].

Die Angaben zum Tabak- und Alkoholkonsum sind in der KiGGS-Basiserhebung über Selbstausfüllfragebögen, in KiGGS Welle 1 dagegen in Telefoninterviews ermittelt worden. In Interviewantworten ist verschiedentlich eine stärkere Tendenz in Richtung sozialer Erwünschtheit beobachtet worden als in Fragebogenantworten [40]. Es kann daher nicht ausgeschlossen werden, dass der beobachtete Rückgang der Prävalenzen zumindest teilweise auf einen entsprechenden Antwort-Bias zurückzuführen ist. Angesichts der guten Übereinstimmung mit Erhebungen, die mit einheitlicher Methodik durchgeführt wurden, wie z. B. die Repräsentativerhebungen der $\mathrm{BZgA}$, dürfte dieser Effekt jedoch gering und damit vernachlässigbar sein.

Eine weitere Limitation der vorliegenden Untersuchung ist in der Erhebung des Alkoholkonsums zu sehen. Während für den Tabakkonsum mit den vorliegenden Daten für ein Monitoring zentrale Indikatoren gebildet werden können und ein Vergleich mit anderen Studien, wie z. B. den Repräsentativerhebungen der BZgA und der ESPAD-Studie des IFT möglich ist, trifft dies auf den Alkoholkonsum nicht bzw. nicht gleichermaßen zu. Allerdings wurde mit dem AUDIT-C in KiGGS Welle 1 ein bewährtes Instrument eingesetzt, das Aussagen über den Risikokonsum und Rauschtrinken ermöglicht. Da geplant ist, den AUDIT-C auch in den nachfolgenden Erhebungen einzusetzen, werden künftig auch Betrachtungen zu zeitlichen Entwicklungen und Trends des Alkoholkonsums bei Jugendlichen möglich sein.

\section{Korrespondenzadresse}

\section{PD Dr. T. Lampert}

Abteilung für Epidemiologie und

Gesundheitsmonitoring

Robert Koch-Institut

General-Pape-Straße 62-64

12101 Berlin

t.lampert@rki.de

\section{Einhaltung der ethischen Richtlinien}

Interessenkonflikt. T. Lampert und B. Kuntz geben an, dass kein Interessenkonflikt besteht.

Dieser Beitrag beinhaltet keine Studien an Menschen oder Tieren.

Finanzierung der Studie. Die Studie wurde mit Mitteln des Robert Koch-Instituts und des Bundesministeriums für Gesundheit finanziert.

\section{Literatur}

1. Drogenbeauftragte der Bundesregierung (2013) Drogen- und Suchtbericht. Mai 2013. Bundesministerium für Gesundheit, Berlin
2. OECD (2012) Health at a Glance: Europe 2012. OECD Publishing, Paris

3. Anderson P, Moller L, Galea G (Hrsg) (2012) Alcohol in the European Union. Consumption, harm and policy approaches. World Health Organization Regional Office for Europe, Copenhagen

4. Mons U (2011) Tabakattributable Mortalität in Deutschland und in den deutschen Bundesländern - Berechnungen mit Daten des Mikrozensus und der Todesursachenstatistik. Gesundheitswesen 73:238-246

5. Bloomfield K, Kraus L, Soyka M (2008) Alkoholkonsum und alkoholbezogene Störungen. Heft 40. In: Robert Koch-Institut (Hrsg) Themenhefte der Gesundheitsberichterstattung des Bundes. RKI, Berlin

6. Adams M, Effertz T (2011) Die volkswirtschaftlichen Kosten des Alkohol- und Nikotinkonsums. In: Singer MV, Batra A, Mann K (Hrsg) Alkohol und Tabak. Grundlagen und Folgeerkrankungen. Thieme, Stuttgart, S 57-62

7. Langness A, Richter M, Hurrelmann K (2005) Gesundheitsverhalten im Jugendalter: Ergebnisse der internationalen „Health Behaviour in School-aged Children"-Studie. Gesundheitswesen 67:422-431

8. Lampert T (2008) Smoking and passive smoking exposure in young people - results of the German health interview and examination survey for children and adolescents (KiGGS). Dtsch Arztebl Int 105:265-271

9. Bundeszentrale für gesundheitliche Aufklärung (2012) Die Drogenaffinität Jugendlicher in der Bundesrepublik Deutschland 2011. Teilband Alkohol. BZgA, Köln

10. U.S. Department of Health and Human Services (2012) Preventing tobacco use among youth and young adults: a report of the surgeon general. U.S. Department of Health and Human Services, Centers for Disease Control and Prevention, National Center for Chronic Disease Prevention and Health Promotion, Office on Smoking and Health, Atlanta

11. Riegg F, Pogarell O (2012) Alkoholmissbrauch und Alkoholabhängigkeit bei Kindern und Jugendlichen in Deutschland. Pädiatrische Praxis 79:137145

12. Breslau N, Fenn N, Peterson EL (1993) Early smoking initiation and nicotine dependence in a cohort of young adults. Drug Alcohol Depend 33:129-137

13. Kendler KS, Myers J, Damaj MI, Chen X (2013) Early smoking onset and risk for subsequent nicotine dependence: a monozygotic co-twin control study. Am J Psychiatry 170:408-413

14. Dawson DA, Goldstein RB, Chou SP, Ruan WJ, Grant $\mathrm{BF}$ (2008) Age at first drink and the first incidence of adult-onset DSM-IV alcohol use disorders. Alcohol Clin Exp Res 32:2149-2160

15. Bundeszentrale für gesundheitliche Aufklärung (2012) Die Drogenaffinität Jugendlicher in der Bundesrepublik Deutschland 2011. Der Konsum von Alkohol, Tabak und illegalen Drogen: aktuelle Verbreitung und Trends. BZgA, Köln

16. Kraus L, Pabst A, Piontek D (2011) Die Europäische Schülerstudie zu Alkohol und anderen Drogen 2011 (ESPAD): Befragung von Schülerinnen und Schülern der 9. und 10. Klasse in Bayern, Berlin, Brandenburg, Mecklenburg-Vorpommern und Thüringen. IFT-Berichte Bd. 181. IFT Institut für Therapieforschung, München

17. Kolip P, Klocke A, Melzer W, Ravens-Sieberer U (Hrsg) (2013) Gesundheit und Gesundheitsverhalten im Geschlechtervergleich. Ergebnisse des WHO-Jugendgesundheitssurvey "Health Behaviour in School-aged Children“. Beltz Juventa, Weinheim Basel 
18. Richter M, Pförtner T-K, Lampert T, HBSC-Team Deutschland (2012) Veränderungen im Tabak-, Alkohol- und Cannabiskonsum von Jugendlichen im Zeitraum von 2002 bis 2010 in Deutschland. Gesundheitswesen 74:42-48

19. Lampert T, Thamm M (2007) Tabak-, Alkohol- und Drogenkonsum von Jugendlichen in Deutschland. Ergebnisse des Kinder- und Jugendgesundheitssurveys (KiGGS). Bundesgesundheitsbl Gesundheitsforsch Gesundheitsschutz 50:600-608

20. Kamtsiuris $P$, Lange M, Schaffrath Rosario A (2007) Der Kinder- und Jugendgesundheitssurvey (KiGGS): Stichprobendesign, Response und Nonresponse-Analyse. Bundesgesundheitsbl Gesundheitsforsch Gesundheitsschutz 50:547-556

21. Kurth BM, Kamtsiuris $P$, Hölling $H$, Schlaud M, Dölle R, Ellert U, Kahl H, Knopf H, Lange M, Mensink GBM, Neuhauser H, Schaffrath Rosario A, Scheidt-Nave C, Schenk L, Schlack R, Stolzenberg H, Thamm M, Thierfelder W, Wolf U (2008) The challenge of comprehensively mapping children's health in a nation-wide health survey: design of the German KiGGS-Study. BMC Public Health 8:196

22. Hölling $H$, Schlack $R$, Kamtsiuris $P$, Butschalowsky H, Schlaud M, Kurth BM (2012) Die KiGGS-Studie. Bundesweit repräsentative Längs- und Querschnittstudie zur Gesundheit von Kindern und Jugendlichen im Rahmen des Gesundheitsmonitorings am Robert Koch-Institut. Bundesgesundheitsbl Gesundheitsforsch Gesundheitsschutz 55:836-842

23. Bush K, Kivlahan DR, McDonell MB, Fihn SD, Bradley KA (1998) The AUDIT alcohol consumption questions (AUDIT-C): an effective brief screening test for problem drinking. Ambulatory Care Quality Improvement Project (ACQUIP). Alcohol Use Disorders Identification Test. Arch Intern Med 158:1789-1795

24. Saunders JB, Aasland OG, Babor TF, de la Fuente JR, Grant M (1993) Development of the alcohol use disorders identification test (AUDIT): WHO collaborative project on early detection of persons with harmful alcohol consumption-II. Addiction 88:791-804

25. Babor TF, Higgins-Biddle JC, Saunders JB, Monteiro MG (2001) AUDIT - The Alcohol Use Disorders Identification Test. Guidelines for use in primary care, 2. Aufl. World Health Organization, Department of Mental Health and Substance Dependence, Geneva

26. Hapke U, von der Lippe E, Gaertner B (2013) Riskanter Alkoholkonsum und Rauschtrinken unter Berücksichtigung von Verletzungen und der Inanspruchnahme alkoholspezifischer medizinischer Beratung. Ergebnisse der Studie zur Gesundheit Erwachsener in Deutschland (DEGS 1). Bundesgesundheitsbl Gesundheitsforsch Gesundheitsschutz 56:809-813

27. Robert Koch-Institut (2011) Daten und Fakten: Ergebnisse der Studie "Gesundheit in Deutschland aktuell 2009“. In: Robert Koch-Institut (Hrsg) Beiträge zur Gesundheitsberichterstattung des Bundes. RKI, Berlin

28. Robert Koch-Institut (2012) Daten und Fakten: Ergebnisse der Studie "Gesundheit in Deutschland aktuell 2010". In: Robert Koch-Institut (Hrsg) Beiträge zur Gesundheitsberichterstattung des Bundes. RKI, Berlin

29. Rumpf HJ, Wohlert T, Freyer-Adam J, Grothues J, Bischof $G$ (2013) Screening questionnaires for problem drinking in adolescents: performance of $A U$ DIT, AUDIT-C, CRAFFT and POSIT. Eur Addict Res 19:121-127
30. Reinert DF, Allen JP (2007) The alcohol use disorders identification test: an update of research findings. Alcohol Clin Exp Res 31:185-199

31. Gual A, Segura L, Contel M, Heather N, Colom J (2002) Audit-3 and audit-4: effectiveness of two short forms of the alcohol use disorders identification test. Alcohol Alcohol 37:591-596

32. Seitz HK, Bühringer G, Mann K (2008) Grenzwerte für den Konsum alkoholischer Getränke: Empfehlungen des wissenschaftlichen Kuratoriums der DHS. In: Deutsche Hauptstelle für Suchtfragen e.V. (Hrsg) Jahrbuch Sucht 2008. Neuland, Geesthacht, S 205-209

33. Lampert T, Müters S, Stolzenberg H, Kroll LE, KiGGS Study Group (2014) Messung des sozioökonomischen Status in der KiGGS-Studie - Erste Folgebefragung (KiGGSWelle 1). Bundesgesundheitsbl Gesundheitsforsch Gesundheitschutz 57. doi: 10.1007/s00103-014-1974-8

34. Lange $M$, Butschalowsky $H$, Jentsch $F$, Kuhnert $R$, Schaffrath-Rosario A, Schlaud M, Kamtsuris $P$, KiGGS-Study Group (2014) Die erste KiGGS-Folgebefragung (KiGGS Welle 1): Studiendurchführung, Stichprobendesign und Response. Bundesgesundheitsbl Gesundheitsforsch Gesundheitschutz 57. doi: 10.1007/s00103-014-1973-9

35. Bundeszentrale für gesundheitliche Aufklärung (BZgA) (Hrsg) (2013) Der Tabakkonsum Jugendlicher und junger Erwachsener in Deutschland 2012. Ergebnisse einer aktuellen Repräsentativbefragung und Trends. BZgA, Köln

36. Deutsches Krebsforschungszentrum (dkfz) (Hrsg) (2009) Tabakatlas Deutschland 2009. dkfz, Heidelberg

37. Lampert T (2013) Tabak - Zahlen und Fakten zum Konsum. In: Deutsche Hauptstelle für Suchtfragen e.V. (Hrsg) Jahrbuch Sucht 2013. Pabst, Lengerich, S 67-90

38. Deutsches Krebsforschungszentrum (dkfz) (Hrsg) (2014) Tabakprävention in Deutschland - was wirkt wirklich? Aus der Wissenschaft - für die Politik. dkfz, Heidelberg

39. Kröger C, Mons U, Klärs G, Orth B, MaschewskySchneider U, Lampert T (2010) Evaluation des Gesundheitsziels „Tabakkonsum reduzieren". Bundesgesundheitsbl Gesundheitsforsch Gesundheitsschutz 53:91-102

40. Kraus L, Piontek D, Pabst A, Gomes de Matos E (2013) Studiendesign und Methodik des Epidemiologischen Suchtsurveys 2012. Sucht 59:309320 